\title{
早期康复护理模式对重症病毒性脑炎患儿肢体功能能力、 吞咽障碍及生活质量的影响
}

Effect of Early Rehabilitation Nursing Model on Limb Function, Dysphagia and Quality of Life in Children with Severe Viral Encephalitis 罗诗婷 刘顺华 卢翔

Shiting Luo Shunhua Liu Xiang Lu

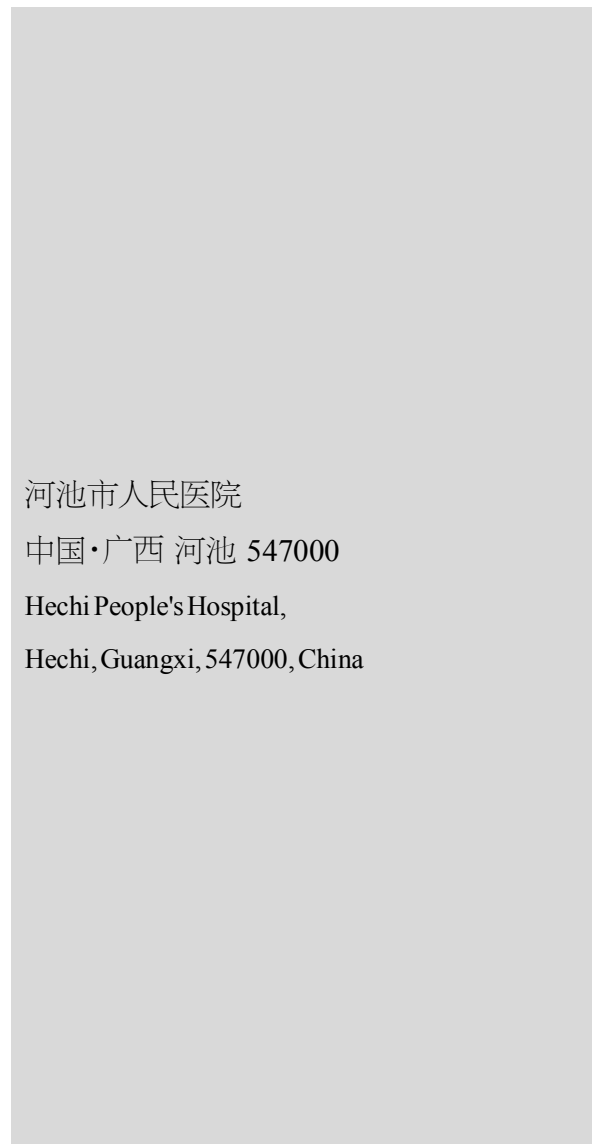

【摘 要】目的: 探究早期康复护理模式对重症病毒性脑炎患儿肢体功能能力、吞咽障碍及 生活质量的影响。方法: 选取我院 94 例重症病毒性脑炎患儿, 随机分为实验组 49 例、对照 组 45 例, 分别予以早期康复护理模式、常规护理模式, 观察护理效果。结果: 实验组肢体功 能、吞咽障碍评分与对照组比较差异具有统计学意义 $(P<0.05)$; 实验组生活质量评分与对 照组比较差异具有统计学意义 $(P<0.05)$ 。结论: 对重症病毒性脑炎患儿予以早期康复护理 模式干预, 能显著改善患儿的肢体运动功能, 减轻吞咽障碍, 提升患儿的生活质量, 值得推 广。

【Abstract】Objective: To explore the effects of early rehabilitation nursing mode on limb functional ability, swallowing disorders and quality of life of children with severe viral encephalitis. Methods: A total of 94 children with severe viral encephalitis in our hospital were randomly divided into experimental group ( 49 cases) and control group ( 45 cases). Results: The differences of limb function and swallowing disorder scores between the experimental group and the control group were statistically significant $(P<0.05)$. The difference of life quality score between the experimental group and the control group was statistically significant $(P<0.05)$. Conclusion: Early rehabilitation nursing mode intervention for children with severe viral encephalitis can significantly improve the limb movement function, reduce swallowing disorders, and improve the quality of life of children, which is worthy of promotion.

【关键词】重症病毒性脑炎; 早期康复护理模式; 肢体功能能力; 吞咽障碍

【Keywords】severe viral encephalitis; early rehabilitation nursing mode; functional ability of limbs; swallowing disorder

【DOI】10.36012/pmr.v2i1.1330

\section{1 引言}

病毒性脑炎为神经内科、儿科常见疾病, 患儿主要表现出 发热、肌痛、咽痛等典型的症状,应当积极予以治疗, 从而减轻 疾病对患儿生长发育的影响[1-2]。值得注意的是,待患儿病情发 展至重症病毒性脑炎时,其往往会伴有不同程度的吞咽障碍, 即摄食困难、构音障碍,同时还会增大营养不良等事件发生的 可能性, 威胁患儿的生命安全, 并且降低患儿的生活质量 ${ }^{[3]}$ 。有 研究表明,在患儿治疗期间,如果能够给予患儿有效的护理配
合, 不仅能改善患儿的吞咽障碍, 还对患儿的肢体运动功能与 生活质量等方面均有积极的作用。因此,除了对患儿进行积极 的药物治疗外, 对患儿进行早期的针对性训练也是很有必要 的。本研究即对我院部分患者应用早期康复护理模式干预, 具 体内容如下。

\section{2 资料与方法}

\section{1 临床资料}

选取 2017 年 3 月至 2020 年 1 月我院 94 例重症病毒性 


\section{创新管理 Innovation Management}

脑炎患儿, 随机分为实验组 49 例、对照组 45 例。实验组中, 男 27 例, 女 22 例, 年龄为 4 12 岁, 平均年龄为 $(6.76 \pm 0.54)$ 岁; 对照组中, 男 22 例, 女 23 例, 年龄为 4 11 岁, 平均年龄为 (6.79 \pm 0.51$)$ 岁。两组基本资料无显著差异 $(P>0.05)$ 。

\section{2 方法}

对对照组应用常规护理模式, 主要内容为用药护理、口腔 卫生护理、饮食护理、口腔训练护理、吞咽护理、头颈部肌肉训 练等常规护理。

对实验组应用早期康复护理模式, 内容如下:第一,建立 早期康复护理小组。组成成员主要包括主治医师、护士长、护 理人员, 并且邀请护理经验较为丰富的医师对组内护理人员 进行培训,培训内容为早期康复护理模式目的、意义, 重症病 毒性脑炎患儿护理要点、步骤等。培训完成后, 护理人员需要 主动询问主治医师患儿的实际病情, 综合评估患儿的肢体运 动功能、吞咽障碍情况, 制定康复护理计划。同时,也要给患儿 家属做好健康教育, 使其了解训练的目的、方法和重要性。第 二,具体实施。首先, 基础训练。对患儿进行咽部冷刺激(于口 腔放置小冰块)、舌肌抗阻训练(外伸舌头)、唇周肌肉训练(按 住患儿头部, 进行口唇按摩)等吞咽训练, 放松患儿的肌肉, 改 善患儿的吞咽障碍症状。其次, 摄食训练。加强患儿的口腔清 洁, 选择患儿较为适宜的体位进食, 减少误吸情况的发生, 饮 食性质依据患儿的恢复情况改变, 从流质食物逐步过渡到半 流质与普食, 饮食按照高蛋白、纤维易消化的原则进行饮食搭 配, 促使患儿更快恢复, 提升患儿的生活质量。再次,语言训 练。护理人员可以使用患儿易于听懂的语言鼓励患儿进行 $a 、$ o、e、1、2、3 等字母、数字的发音练习, 有利于提升患儿的口腔 与声带运动功能, 从而促使患儿的语言功能尽快恢复。这样的 训练对于患儿的吞咽能力也有很大的好处。最后, 肢体康复训 练。每日鼓励患儿进行肢体与各关节的运动, 如从肩关节、肘 关节、腕关节、膝关节、踝关节等运动, 幅度依据患儿的实际耐 受情况逐渐增大。针对痉孪期患儿, 护理人员可鼓励其手握柱 形训练物, 进行屈伸上肢训练, 训练结束后, 护理人员对其进 行按摩, 避免发生肌张力过度的情况, 防止增加患儿的不适, 促使患儿的肢体运动功能更快恢复。

\section{3 疗效判定 / 观察指标}

\subsection{1 两组肢体功能、吞咽障碍评分}

采用 Fugl-Meyer 表对患儿的肢体运动功能进行评定, 满 分 100 分, 分值越高, 说明患儿的肢体运动功能越好。采用DDS 吞咽障碍调查问卷量表评估患儿的吞咽障碍情况, 分值越高, 说明患儿的吞咽障碍越严重。

\subsection{2 两组生活质量评分}

采用我院 SF-36 生活质量评估量表, 对患儿的社会角色 和健康状况以及认知功能等方面进行评分, 满分 100 分, 分值 越高, 则代表患儿的生活质量越好。

\section{4 统计学分析}

利用 SPSS22.0 软件分析数据, $(\bar{x} \pm s)$ 表示计量资料, 采用 $t$ 检验、 $\chi^{2}$ 对比, $P<0.05$ 表示差异显著。

\section{3 结果}

\section{1 两组肢体功能、吞咽障碍评分比较}

实验组肢体功能、吞咽障碍评分与对照组比较差异具有 统计学意义 $(P<0.05)$, 具体如表 1 所示:

表 1 两组肢体功能、吞咽障碍评分比较

\begin{tabular}{c|c|c|c}
\hline 组别 & 例数/例 & 肢体功能/分 & 吞咽障碍/分 \\
\hline 实验组 & 49 & $78.54 \pm 2.32$ & $5.76 \pm 0.06$ \\
\hline 对照组 & 45 & $60.87 \pm 4.55$ & $7.65 \pm 1.08$ \\
\hline$t$ & - & 24.006 & 12.335 \\
\hline$P$ & - & 0.000 & 0.000 \\
\hline
\end{tabular}

\section{2 两组生活质量评分比较}

实验组生活质量评分与对照组比较差异具有统计学意义 $(P<0.05)$, 具体如表 2 所示:

表 2 两组生活质量评分比

\begin{tabular}{c|c|c}
\hline 组别 & 例数/例 & 生活质量评分/分 \\
\hline 实验组 & 49 & $89.87 \pm 4.09$ \\
\hline 对照组 & 45 & $78.76 \pm 3.31$ \\
\hline$t$ & - & 14.398 \\
\hline$P$ & - & 0.000 \\
\hline
\end{tabular}

\section{4 讨论}

重症病毒性脑炎为小儿危重疾病,积极进行治疗的同时,还 应当配合有效护理干预,从而改善患儿运动障碍、吞咽障碍等后 遗症, 提升患儿的生活质量, 促使患儿能更好地发育成长 ${ }^{[4]}$ 。

本次研究, 即对我院实验组患儿应用早期康复护理模式 干预, 对对照组患儿应用常规护理模式干预 ${ }^{[5]}$ 。通过观察护理 效果发现, 实验组在肢体功能、吞咽障碍评分, 生活质量评分 上均较对照组更优 $(P<0.05)$ 。早期康复护理模式是依据患儿 的实际病情制定的有效康复训练方案, 意在通过早期、由浅人 深的康复护理, 改善患儿的病情症状, 促使患儿更快地康复, 减轻患儿的痛苦。而常规护理更注重对患儿进行疾病护理, 对 于患儿康复后能否独立生存这方面并未有所考虑, 早期康复 护理模式正好能够弥补这一缺点, 在减轻患儿痛苦的同时, 能

(下转第 44 页) 
创新管理 Innovation Management

\section{1 .2 对患者的评估有待加强}

护理人员对患者的评估还有待加强, 没有意识到患者自 行拔掉管道的高危因素, 进而没有采取相应措施进行预防, 无 形中也加重了管道护理不良事件对患者的伤害程度。

\subsection{3 和患者的沟通有待加强}

第一,由于护理人员和患者的沟通不及时,使得护理人员 对患者的病情和心理状态等不够了解, 难以在患者拔掉管道 后第一时间发现并进行处理; 第二,护理人员仅仅是简单交代 了管道留置的注意事项，对于自行拔掉管道的危害没有进行 强调,使得患者认知不清,进而自行拔管。

\section{1 .4 管道固定得不够牢}

例如, 在本次研究的 15 起管道护理不良事件中，就有 5 起是因为管道固定不牢而导致的管道滑脱，在很大程度上也 给患者的治疗效果和身体健康造成了一定影响。

\section{2 管道护理不良事件发生的主要科室和对象}

第一, 在实际的治疗过程中, 危重症患者的病症情况变化 得较快, 而且患者的生命一般情况下也是依赖于多条管道进 行维持的, 因此一旦出现管道护理不良事件, 对患者的伤害也 较大; 第二, 对于一些老年患者, 其在接受治疗的过程中, 生理 机能经常会出现退行性的变化和相关疾病, 因此患者在接受 健康指导方面, 能力较弱, 记忆力也较弱。

\section{(上接第 42 页)}

够有效改善患儿的预后情况,提升患儿的生活质量。早期康复 护理模式针对各个影响患儿的并发症，制定简单有效的康复 训练, 对患儿进行早期的、由浅及深的康复护理干预。从护理 内容看, 首先是建立早期康复护理小组,有利于全面了解患儿 的病情, 制定符合患儿的实际护理需求的康复训练计划。之后 进入具体实施阶段, 从基础训练、摄食训练、语言训练、肢体康 复训练等方面对患儿进行护理，有效改善患儿的吞咽障碍症 状, 减少或杜绝营养不良事件的发生, 提升患儿的肢体运动功 能,并提升患儿的生活质量。早期康复护理模式干预有效地改 善了患儿的肢体功能、吞咽能力, 从而大大地提高了患儿的生 活质量,为患儿回归社会做好了准备。

\section{5 结语}

综上所述，对重症病毒性脑炎患儿予以早期康复护理模

\section{3 管道护理不良事件的解决对策}

\subsection{1 加强对护理人员的培训}

第一, 医院要定期组织护理人员进行护理案件的分析, 通 过实际的案件让护理人员明确在管道护理工作中的一些细节 问题,并在日常的护理工作中进行注意; 第二,各个护理人员 之间也要定期进行交流, 做到资源共享, 对常见的管道护理风 险,护理人员可通过相互交流研究预防措施; 第三,在进行护 理工作交接时,护理人员需检查患者的管道固定是否牢固,注 意落实护理人员的责任,不断提高护理人员的安全防范意识。

\section{3 .2 建立并完善管理制度}

医院需要建立并完善管道护理管理制度, 成立专门的管 理小组, 以此来对管道护理工作进行监督和管理。另外, 医院 还需要建立并完善管道滑脱预防制度, 加强对护理人员的管 道护理指导, 并进行管道滑脱危险程度的划分, 帮助护理人员 对不同的患者进行有针对性的护理。

\subsection{3 加强安全教育}

对于重点人群, 如危重症患者、老年患者, 护理人员需要 加强安全教育, 提高患者的风险意识,避免其在治疗和护理工 作中自行拔掉管道,引发不良事故。

\section{参考文献}

[1]石慧.管道护理不良事件的原因分析及其对策 [J].中国当代医 药,2015(29):176-178.

式干预, 能显著改善患儿的肢体运动功能, 促进肢体功能能力 的恢复, 提高吞咽能力, 改善患儿的生活质量, 值得推广。

\section{参考文献}

[1]钟顺平,陈建江,陈丞浦.119 例小儿病毒性脑炎临床分析[J].中 国实用神经疾病杂志,2017,20(22):83-85.

[2]李永春, 李小芹,张迎辉.儿童肠道病毒性脑炎的临床特点及脑 脊液病毒检测结果分析 [J].安徽医药,2019,23(7):1369-1372.

[3] 吴蕾. 连续性血液净化技术在重症病毒性脑炎中的应用研究 [J].中国血液净化,2017,16(3):176-178.

[4]朱晓霞.纳洛酮及丙种球蛋白治疗小儿重症病毒性脑炎的临床 疗效及安全性分析[J].中国实用医药,2019(24):1-3.

[5]吴俊,贾秀英.延续性护理对重症病毒性脑炎后遗症儿童生存 质量的影响[J].贵州医科大学学报,2017,42(2):244-246. 